\title{
Fearful symmetry: Subversion of asymmetric division in cancer development and progression
}

\author{
Jeevisha Bajaj ${ }^{1,2,3}$, Bryan Zimdahl ${ }^{1,2,3}$, and Tannishtha Reya ${ }^{1,2,3,{ }^{*}}$ \\ ${ }^{1}$ Department of Pharmacology, University of California San Diego School of Medicine La Jolla, \\ $\mathrm{CA}$ \\ ${ }^{2}$ Sanford Consortium for Regenerative Medicine, La Jolla, CA \\ ${ }^{3}$ Moores Cancer Center, University of California San Diego School of Medicine, La Jolla, CA
}

\begin{abstract}
Asymmetric division is an evolutionarily conserved process that generates daughter cells with different fates through the unequal partitioning of fate determinants. While asymmetric division is particularly important in generating diversity during development, its dysregulation can also promote oncogenesis. In particular, signals that shift the normal balance of symmetric and asymmetric division can lead to a differentiation arrest and trigger cancer progression. Here we discuss the studies that have provided increasing support for this idea: beginning with original work carried out in Drosophila, we trace more recent data in mammalian systems that suggest that the subversion of asymmetric division can contribute significantly to the development and progression of both hematologic malignancies and solid cancers.
\end{abstract}

\section{Introduction}

During embryonic development and in adult tissues, stem cells divide to give rise to cells with distinct fates to generate the diversity of cells needed for normal tissue functioning. While extrinsic cues can play an important role in controlling differentiation, intrinsic cues can also act through asymmetric division to control the birth of differentiated daughter cells. Asymmetric cell division involves the unequal segregation of proteins or RNA that can direct distinct programs to influence the fate of the cell. Stem cells that undergo asymmetric division yield one daughter that remains a stem cell and another that becomes differentiated. By contrast symmetric divisions- either symmetric renewal or symmetric differentiationgenerate daughter cells with identical fate (Figure 1). Often used as a mechanism for diversification during development, more recent work suggests that asymmetric division may also be integral to cancer establishment and progression. In this review we give a brief overview of the molecular mechanisms of asymmetric division primarily identified through research in invertebrates, and focus thereafter on its emerging function in mammalian development and oncogenesis.

\footnotetext{
*To whom correspondence should be addressed.
} 


\section{Asymmetric Division in Model Organisms}

Studies with Drosophila and adult mammalian stem cells have identified at least three distinct classes of proteins involved in asymmetric division. These include Polarity proteins such as Partitioning defective 3 (Par3), Par6, and atypical Protein Kinase C (aPKC), regulators of spindle orientation such as Pins, Disc large (Dlg), Inscuteable (Insc) and Lis1, and fate determinants such as Numb, Prospero/Prox1, Brain tumor (Brat) and Musashi (Msi). Drosophila neural development provides a classic example of cells undergoing asymmetric division. Here, the dividing neuroblasts are polarized, with the polarity proteins (such as Pars) migrating to the apical membrane. This apical polarization is required for the transient accumulation of fate determinants such as Numb, Prospero (Pros) and Brat, and adaptor proteins such as Miranda, at the basal membrane. This is driven by a cascade of phosphorylation events triggered by the kinase Aurora A and eventually results in the inactivation of $\mathrm{Lgl}$ (Lethal 2) giant larva) by aPKC at the apical neuroblast cortex, allowing it to be replaced by Par3 at the apical complex. This now permits aPKC to phosphorylate Numb and thus release it from the apical cortex, allowing accumulation on the opposite, basal side of the cell (1). Like Numb, Miranda is also phosphorylated by aPKC and localized to the basal cortex. Miranda in turn binds to Pros and promotes its basal localization. Once inherited by the differentiated daughter cell, Numb represses Notch signaling and the transcriptional activity of Pros promotes a differentiated state (2). In addition to the initiation of polarity, the proper orientation of the mitotic spindle is also essential for correct partitioning of the fate determinants and is regulated by the Ga-Pins-Mud pathway or the Pins-Dlg-Khc73 pathway. The former involves apical localization of the adaptor protein Insc and the recruitment of Pins and mushroom body defective (Mud), which interact with the dynein-dynactin complex, including Lis1. The recruitment of the dynein-dynactin complex to the apical cortex generates a pulling force to lock one centrosome at the apical pole, which subsequently leads to the proper alignment of the mitotic spindle along the apical/basal polarity axis. The second spindle orientation pathway requires Pins, Dlg and its interaction partner Khc-73, a microtubule plus-end-directed kinesin motor heavy chain (3). Implementation of this pathway involves the localization of Khc73 to microtubule plus-ends where it binds to Dlg, which associate with Pins to facilitate cortical microtubule anchoring and subsequent stabilization of the mitotic spindle (3).

Outside signals can also trigger spindle orientation changes as shown in the developing $C$. elegans embryo and in the Drosophila germ cell niche. In the C. elegans embryo, the posterior signaling cell (P2) secretes Wnt ligands that interact with Frizzled on the adjacent Endoderm Mesoderm founder cell (EMS), such that the spindle in the EMS actively rotates to the point of contact with $\mathrm{P} 2$ and sets up an asymmetric axis. This polarization ensures that the cell born next to P2 adopts an endodermal fate, while the one dividing away adopts a mesodermal fate (4). Similarly, the Drosophila gonadal stem cell niche expresses ligands that can activate either JAK/STAT signaling (in males) or TGF $\beta$ signaling (in females) in the adjacent stem cell, thus promoting self-renewal. Since these signals act over shortdistances, cells that divide away from the niche differentiate, while those directly adjacent to the niche cell remain stem-like(5). 


\section{Asymmetric Division in Mammalian Development}

Although the concepts of asymmetric division were initially developed and demonstrated in invertebrates such as Drosophila and C. elegans, over the last decade it has become clear that this can be an integral mechanism for diversification in mammalian development as well (6). For instance, time lapse imaging studies of the developing mouse cortex have shown that the orientation of cleavage furrow can determine the fate of daughter cells (7); the fact that isolated cortical progenitor cells only divide in a stereotyped manner (8) suggest that their division may be independent of external cues. Stratification of the epidermis as well results from asymmetric divisions, where the orientation of the spindle in the dividing basal layer cells can determine the fate of the progeny. Experiments using GFP-labeled centrin have shown that a perpendicular alignment of the spindle gives rise to progeny with distinct fates, wherein the basal cell remains stem-like and the suprabasally dividing cell differentiates (9).

Asymmetric division can also lead to cellular diversification, and preservation of the stem cell state during normal hematopoiesis. Based on a screen of asymmetric division mediators, studies have shown that shRNA mediated knockdown of the polarity proteins such as Pard6a and Prkcz (aPKC homolog) in hematopoietic stem cells (HSC) can lead to increased differentiation and loss of HSC repopulation, and knocking-down the fate determinant Prox 1 promoted increased accumulation of immature and mature cells (10). Similarly, genetic loss of function of the spindle orientation regulator Llgll (11) in the hematopoietic tissue led to enhanced engraftment and better HSC repopulation capacity due to increased proliferation. While these studies did not specifically track asymmetric segregation of fate determinants, the adaptor protein complex associated Ap2a2 protein has been shown to segregate asymmetrically during HSC division in vitro, with its overexpression triggering an in vivo proliferative advantage (12). However, the significance of Ap2a2 and Numb segregation being discordant, and whether it indicates a unique mechanism of control over symmetric renewal is not clear.

There is also genetic evidence that asymmetric division can influence hematopoietic stem cell function in vivo. The findings that Numb, which can trigger differentiation, is segregated and inherited differentially into two daughter cells (13), led to work showing that the Numb inhibitor Musashi (Msi) was highly expressed in stem cells, and lost during differentiation. Genetic deletion of Msi2 led to reduced frequency of stem cells (14); this was confirmed in independent studies, which demonstrated that deletion of Msi2 causes HSC defect in the context of transplantation (15). While Msi serves as a fate determinant, another mechanism by which asymmetric division may be regulated is through the orientation of the spindle during division. Spindle orientation can be critical in specifying the plane of division and the equal or unequal inheritance of fate determinants into two daughter cells, thus controlling differentiation state. Supporting this possibility is the finding that deletion of the dynein binding protein Lis1, which is critical for correct spindle positioning, leads to depletion of HSCs and a dramatic bloodless phenotype, and that these striking phenotypes are related to defects in spindle anchoring and asymmetric division (16). 


\section{Asymmetric Division in Cancer}

Over the past few decades, cancer research has placed a heavy emphasis on understanding aberrant proliferation and survival as key drivers of oncogenesis. However proliferation and survival alone are not sufficient to endow cancer with all its malignant properties, and therapies such as radiation and chemotherapy that solely target these attributes can have limited impact. An important element of cancer is its striking structural similarities with development: cancers can be heterogeneous and utilize self-renewing mechanisms for propagation, and cancers often reverse differentiation to drive progression (17). In these contexts the subversion of asymmetric division could be of great significance. Thus, asymmetric division could enable generation of diversity within a cancer, while protecting a core population that harbors aberrant self-renewal, and a shift in the balance of asymmetric and symmetric divisions can trigger arrested differentiation and progression. Because of its potentially powerful influence, it is critical to understand how asymmetric division may contribute to oncogenesis, and whether disruption of this process may be of therapeutic value.

Pioneering work using genetic screens in Drosophila were the first to demonstrate that mutations in several genes involved in asymmetric cell division of neuroblasts (18) such as $l g l$ and brat (19) can trigger the development of brain tumors. Further, loss of function of prospero (20) or numb (21) could phenocopy brat mutations. These studies indicated that defects in the function of fate determinants and regulators of asymmetric division could lead to uncontrolled symmetric renewal of neuroblasts and the development of transplantable tumors. Although these observations suggested that loss of asymmetric regulation can induce immortalization in neural stem cells and may be an initiating event in oncogenesis, the relevance of these discoveries for mammalian oncogenesis remained unclear till very recently.

\section{Asymmetric division in hematologic malignancies}

The studies in hematopoietic stem cells suggest an emerging picture where pathways associated with asymmetric division can influence mammalian hematopoietic stem cell fate and function. The impact of asymmetric division in hematological malignancies was beginning to be explored around the same time, using leukemias as a model system. Much of the work to date has been on myeloid leukemia, with the influence of asymmetric division explored first in context of progression towards the acute phase leukemia associated with oncogenes BCR-ABL and NUP98-HOXA9 (13). Visualization of division patterns using real time imaging of Notch reporter activity (used as a surrogate for the immature state) indicated that expression of $\mathrm{BCR}-\mathrm{ABL}$, the translocation which drives chronic myelogenous leukemia (CML), does not alter the normal balance between symmetric and asymmetric divisions. However expression of NUP98-HOXA9, which is linked to blast crisis CML and some de novo AMLs, shifted the balance away from asymmetric divisions to symmetric renewal divisions. Interestingly, loss of the tumor-suppressor Pml (Promyelocytic leukemia protein) has been shown to promote symmetric commitment divisions of normal HSCs (22). Although it remains to be tested, it is interesting to speculate that the exhaustion of CML 
stem cells (23) in Pml-deficient mice, could be a result of increasing differentiation over multiple cell division cycles.

The studies above suggest that increased symmetric renewal division could form the basis of the differentiation arrest that occurs in progression, and that modulation of signals that increase asymmetry may enable imposition of a more differentiated state and resolution of disease. This possibility drove exploration of determinants that may be altered in oncogenesis and may be mediators of increased self-renewal and progression. While Numb is downregulated as CML progresses to blast crisis, Musashi, an RNA binding protein and Numb repressor is strikingly upregulated (14). Further, genetic deletion of Msi2 or ectopic expression of Numb significantly impairs in vivo leukemia progression in a mouse model of blast-crisis CML (14). AML cell lines also express Msi2 suggesting that there is a general pattern of Msi upregulation in high-grade hematologic malignancies (24). These experiments suggest that increased expression of Msi2 in part through suppression of Numb may impair asymmetric division and arrest differentiation thus leading disease progression Imaging studies showing that Numb overexpression impose more asymmetric divisions supported this link (Lento and Reya, unpublished observations).

While protein determinant play a direct role in specifying fate, the correct partitioning of these determinants, usually controlled by precise spindle orientation can also serve as a point of control in oncogenesis. For instance, acute myeloid leukemias have lowered expression of Llgl1 than lower grade myeloproliferative neoplasms and loss of Llgll expression is associated with poor survival in AML (11). Functionally the possibility of incorrect spindle orientation promoting leukemic growth has recently been tested in CML and AML leukemic growth in the context of the dynein-binding protein Lis1 (16). Loss of Lis1 expression was shown to not only result in a profound bloodless phenotype in the developing mouse embryo, but also lead to striking defects in hematopoietic stem cell differentiation. Moreover, Lis1 loss nearly resolves the in vivo progression of aggressive myeloid disease. Mechanistically, the loss of Lis1 leads to randomization of spindle orientation, which in turn promotes a marked increase in the frequency of asymmetric divisions, as scored by Numb inheritance in the progeny of dividing leukemic stem cells. Thus, Lis1 loss is accompanied with an increase in leukemic differentiation, which may be the underlying reason why the disease fails to establish or propagate in the absence of Lis1. Thus, these data raise the possibility that the enforced asymmetric division of leukemic cells may promote differentiation and serve as a method to resolve aggressive myeloid disease.

\section{Asymmetric Division in Solid Cancers}

While mutations in many genes regulating asymmetric division of normal stem cells, such as $L G L$, atypical $P K C \zeta$, SCRIB1, DLG, MSI1 etc., have been associated with solid tumors (25), only some studies demonstrate an aberrant increase in symmetric renewal by directly visualizing the inheritance of fate determinants in murine and human cancers. However, since most of this work is done using established tumor cells, it chiefly demonstrates the effect of loss of asymmetric division in the context of continual propagation of an established tumor and does not address the role of asymmetric division in tumor initiation or metastasis. They primarily fall in three categories: those implicating the loss of Numb 
regulation through tumor suppressors, those implicating microRNA mediated regulation of Notch/Numb, and studies describing asymmetric division independent of stem cell or differentiation state.

Loss-of-function of the tumor-suppressor gene TP53 has not only been shown to promote tumorigenesis and epithelial-to-mesenchymal transition in solid cancers (26), but has also been shown to promote maintenance of the stem cell state (27). Early evidence for a role of p53 in stem cell divisions came from work on normal and malignant mammary sphere cultures that enrich for stem cell populations (28). PKH fluorescent dye labeling of these spheres showed that the loss of p53 activity, either genetic or under the influence of the Erbb2 oncogene, can induce a shift from asymmetric to predominantly symmetric renewal divisions. While these experiments, coupled with limiting dilution assays, show an increase in the functional stem cell populations following p53 loss, they do not rule out the possibility of emergence of progenitor cells with limited proliferation capacity. This is especially true since the PKH staining is not accompanied with analysis of cancer stem cell markers. Since the increase in symmetric divisions is scored by PKH dye-dilution and symmetric inheritance of Numb, it is possible that many of the cells that proliferated and arose in vitro were committed progenitors with high Numb levels. This would be consistent with the observation that the highest tumor-initiating ability resided in the non-dividing $\mathrm{PKH}^{\text {hi }}$ cells, while the $\mathrm{PKH}^{\mathrm{mid}}$ and $\mathrm{PKH}^{\text {lo }}$ cells had significantly lower numbers of cancer stem cells. Nevertheless, this study does provide conclusive evidence for a role for $\mathrm{p} 53$ in regulating the numbers of stem/progenitor cells in mammary tissues.

Experiments using paired-cell assays have also implicated the loss of p53 in promoting selfrenewal of oligodendrocytic progenitors (OPCs) and oligodendrogliomas that arise from these cells (29). The paired-cell or clone-splitting assay was originally developed to quantitate asymmetric vs. symmetric divisions in context of hematopoietic stem cells (30). Here, single cells were allowed to undergo one round of mitosis, physically separated and functionally evaluated for their ability to undergo multi-lineage differentiation. This technique was subsequently modified in the context of neural stem cells, where the daughter cell "pairs"were stained for fate-determinants, which served as a surrogate for functional analysis (31). Using this assay, it has been shown that the neural/glial antigen 2 (NG2) segregates asymmetrically in about $50 \%$ of dividing OPCs, and co-segregates with EGFR (29). However, in a mouse model of oligodendroglioma ( $100 \beta$-verB; $\left.p 53^{+/-}\right)$, there was a marked reduction in asymmetrically dividing cells with a concomitant increase in symmetric renewal and in the total number of OPCs. Interestingly, the homozygous loss of p53 led to a reduction in asymmetric division of normal OPCs and enhanced symmetric renewal. While the molecular mechanisms regulating the shift between asymmetric to symmetric divisions in malignant oligodendrocytic progenitors are yet to be defined, these data suggest that the loss of p53 may play an active role in this process, as seen earlier in mammary cells.

Recent work has shown that the expression of Trim3 (Tripartite Motif Containing 3) protein, the mammalian homolog of Drosophila brat, is markedly reduced in brain tumors in comparison to normal brain (32). Moreover, genetic loss of TRIM3 is significantly associated with TP53 mutations. Interestingly, the enforced expression of Trim3 led to a marked decrease in Nestin ${ }^{+}$cells in glioblastoma neurospheres, suggesting that its loss can 
switch the balance from asymmetric division to symmetric renewal. Mechanistically, high Trim3 not only suppressed MYC but also correlated with low Msi, high Numb and low Notch. These observations suggest that fate determinants other than Numb may also act in conjunction with p53 to regulate asymmetric division.

Two recent studies suggest that microRNAs may regulate asymmetric division of cells in human colon cancer derived sphere cultures that enrich for cancer stem cell-like populations. The first of these showed that an important p53 target micro-RNA, Mir34a (33), acts as a novel fate determinant in established colon cancer cultures in vitro (34). Using pair-cell assays, Mir34a was found to asymmetrically segregate into the differentiating cell, and not the stem cell-like progeny which retained ALDH1 expression. Consistent with a prodifferentiation function, knocking-down Mir-34a expression resulted in a marked reduction in asymmetric division and a concomitant rise in symmetric renewal divisions. As a possible downstream mechanism, Mir34a was shown to bind to and sequester Notch1 mRNA and prevent its translation, thus promoting differentiation. Interestingly, unlike Mir34a, Numb did not consistently segregate away from ALDH1 in asymmetrically dividing cells. This indicates that in colon cancer cells, in vitro asymmetric division and reduced Notch activity can be controlled by mechanisms independent of Numb, but dependent on p53.

Given that Numb is known to stabilize p53 (35), the studies described in this section, along with experiments showing the necessity for $\mathrm{p} 53$ in Numb dependent differentiation of CML (14), raise the possibility that the Numb/Notch/p53/Mir-34a axis may promote differentiation in a wide range of normal and malignant stem cell populations.

A second study on colon cancer and micro-RNAs used long-term colon cancer spherecultures to show that Snail-dependent activation of Mir146a is necessary for symmetric renewal of CSCs in established cancers (36). These experiments have shown that nuclear Snail, nuclear $\beta$-catenin and Mir146a co-segregate with the stem cell marker CD44 in the small fraction of asymmetrically dividing sphere cells. Mechanistically, Snail expression was shown to promote nuclear translocation of beta-catenin, which in turn transcriptionally activated Mir146a. Moreover, it was shown that Mir146a directly binds to 3'-UTR of Numb and represses it. Interestingly, in this system, Numb was shown to directly bind $\beta$-catenin and affect its stability. This suggests that Numb can promote differentiation not only by inhibiting the Notch pathway but by also affecting canonical Wnt signaling, which is known to promote the stem cell state in many systems (37). While these studies identify exciting new regulators of asymmetric division in cancers, it is critical to test if the mechanisms proposed here are active, and mediate cancer progression in vivo.

Recent work using cancer cell lines suggests that other types of asymmetry may exist and be relevant to therapeutic strategies in solid cancers. Specifically, studies of long established human breast and colon cancer cell lines have shown that a small fraction of cells that express high levels of AKT can give rise to G0-like cells that are $\mathrm{AKT}^{\mathrm{lo}} \mathrm{Ros}^{\mathrm{lo}} \mathrm{Hes} \mathrm{1}^{\mathrm{hi}}$ (38). While this population does not express known cancer stem cell markers, it does show enhanced resistance to chemotherapy. Interestingly, a similar $\mathrm{AKT}^{\mathrm{lo}} \mathrm{Hes} 1^{\text {hi }}$ population of cells is part of the residual disease in patients treated with chemotherapy. Although the ability to evade chemotherapy is an attribute of cancer stem cells (39), it is not clear if the 
G0-like cells seen in these cultures also have features of enhanced tumorigenicity, or if the cells that remain after therapy in patients have the capacity to re-initiate tumors. It is provocative to hypothesize that since these G0-like cells show enhanced activation of the Notch target gene Hes l, this may mark them as having a more stem cell like phenotype and function. However, without transplantation studies, it is difficult to assess the functional contribution of these cells. Further, the changes in Notch signaling may be a consequence of low Numb expression; however this was not directly examined in the study. Thus, this work, along with earlier studies on glioma, breast cancers (40) and pleural mesothelioma (41), raises the possibility that enhanced Numb expression, or loss of Notch signaling, not only promote differentiation but also sensitize cells to standard chemo- and radiotherapy.

\section{Future Perspectives}

Work over the past decade has shown that asymmetric division may be critical not only during homeostasis, but may also be essential for controlling oncogenic progression. However, it is important to note that while most studies have shown that loss of fate determinants or asymmetric division regulators can promote cancer progression and expansion of malignant cells, not every work has mapped the inheritance of fatedeterminants to directly demonstrate a shift in division patterns from asymmetric division to symmetric renewal.

Nevertheless, while much has been done in terms of identifying mutations that promote expansion of malignant stem cells and aggressive disease, and many different mechanisms initiating asymmetric divisions have been identified, most studies point towards a downstream deregulation of the Notch/Numb/p53 axis. It is also likely that miR34a, a major p53 target microRNA, regulates asymmetric division downstream of p53 in multiple cancers. However, the function of many other regulators of asymmetric division in cancer remains unknown. Similarly, it is also not clear if other developmental pathways involved in maintaining the stem cell state in Drosophila germ cell niche or in normal adult stem cells, such as JAK/STAT signaling, TGF $\beta$ and Hedgehog, are key players in many malignancies may exert their influence in part by specifying division patterns in cancer.

Despite the progress made in understanding the role of asymmetric division in cancer development, many areas of investigation remain open. For instance, it remains to be determined if mutations in the regulators of asymmetric division can be tumor-initiating events in mammalian cancers, or if their expression only changes as a downstream consequence of other oncogenic drivers. Moreover, it is likely that asymmetric division contributes at distinct stages of tumor development and as further evidence accumulates, it may become clearer if specific classes of signals regulate different oncogenic events. Moreover, the extrinsic or intrinsic signals that determine how and when a cancer stem cell will symmetrically renew or undergo asymmetric division to give rise to other tumor cell types have not been identified. Since the niche of a normal stem cell is known to play an important role in determining the fate of daughter cells (5), the influence of the tumor microenvironment on dividing cancer stem cells is an area worth exploring. 
Current treatment strategies such as radiation and chemotherapy primarily target proliferation and survival, and can often have limited impact. The work we discuss here brings into focus how aberrant shifts from asymmetric divisions towards symmetric divisions can trigger a 'fearful symmetry' (42) that drives cancer development and progression. These emerging ideas also raise the possibility that regulators of asymmetric division could be modulated to shift the balance back towards increased asymmetry and may thus serve as a new class of targets in cancer therapy.

\section{Acknowledgments}

J.B. is supported by a postdoctoral fellowship from the National Cancer Center and B.Z. received support from US National Institutes of Health (NIH) Cancer Biology Training Grant (T32 CA 59365-18).

\section{References}

1. Wirtz-Peitz F, Nishimura T, Knoblich JA. Linking cell cycle to asymmetric division: Aurora-A phosphorylates the Par complex to regulate Numb localization. Cell. 2008; 135(1):161-73. [PubMed: 18854163]

2. Knoblich JA. Mechanisms of asymmetric stem cell division. Cell. 2008; 132(4):583-97. [PubMed: 18295577]

3. Siegrist SE, Doe CQ. Microtubule-induced Pins/Galphai cortical polarity in Drosophila neuroblasts. Cell. 2005; 123(7):1323-35. [PubMed: 16377571]

4. Goldstein B. Cell contacts orient some cell division axes in the Caenorhabditis elegans embryo. The Journal of cell biology. 1995; 129(4):1071-80. [PubMed: 7744956]

5. Yamashita YM, Fuller MT, Jones DL. Signaling in stem cell niches: lessons from the Drosophila germline. Journal of cell science. 2005; 118(Pt 4):665-72. [PubMed: 15701923]

6. Knoblich JA. Asymmetric cell division during animal development. Nature reviews Molecular cell biology. 2001; 2(1):11-20. [PubMed: 11413461]

7. Chenn A, McConnell SK. Cleavage orientation and the asymmetric inheritance of Notch1 immunoreactivity in mammalian neurogenesis. Cell. 1995; 82(4):631-41. [PubMed: 7664342]

8. Qian X, Goderie SK, Shen Q, Stern JH, Temple S. Intrinsic programs of patterned cell lineages in isolated vertebrate CNS ventricular zone cells. Development. 1998; 125(16):3143-52. [PubMed: 9671587]

9. Lechler T, Fuchs E. Asymmetric cell divisions promote stratification and differentiation of mammalian skin. Nature. 2005; 437(7056):275-80. [PubMed: 16094321]

10. Hope KJ, Cellot S, Ting SB, MacRae T, Mayotte N, Iscove NN, et al. An RNAi screen identifies Msi2 and Prox1 as having opposite roles in the regulation of hematopoietic stem cell activity. Cell stem cell. 2010; 7(1):101-13. [PubMed: 20621054]

11. Heidel FH, Bullinger L, Arreba-Tutusaus P, Wang Z, Gaebel J, Hirt C, et al. The cell fate determinant Llgl1 influences HSC fitness and prognosis in AML. The Journal of experimental medicine. 2013; 210(1):15-22. [PubMed: 23277453]

12. Ting SB, Deneault E, Hope K, Cellot S, Chagraoui J, Mayotte N, et al. Asymmetric segregation and self-renewal of hematopoietic stem and progenitor cells with endocytic Ap2a2. Blood. 2012; 119(11):2510-22. [PubMed: 22174158]

13. Wu M, Kwon HY, Rattis F, Blum J, Zhao C, Ashkenazi R, et al. Imaging hematopoietic precursor division in real time. Cell stem cell. 2007; 1(5):541-54. [PubMed: 18345353]

14. Ito T, Kwon HY, Zimdahl B, Congdon KL, Blum J, Lento WE, et al. Regulation of myeloid leukaemia by the cell-fate determinant Musashi. Nature. 2010; 466(7307):765-8. [PubMed: 20639863]

15. de Andres-Aguayo L, Varas F, Kallin EM, Infante JF, Wurst W, Floss T, et al. Musashi 2 is a regulator of the HSC compartment identified by a retroviral insertion screen and knockout mice. Blood. 2011; 118(3):554-64. [PubMed: 21613258] 
16. Zimdahl B, Ito T, Blevins A, Bajaj J, Konuma T, Weeks J, et al. Lis1 regulates asymmetric division in hematopoietic stem cells and in leukemia. Nature genetics. 2014; 46(3):245-52. [PubMed: 24487275]

17. Reya T, Morrison SJ, Clarke MF, Weissman IL. Stem cells, cancer, and cancer stem cells. Nature. 2001; 414(6859):105-11. [PubMed: 11689955]

18. Gateff E. Malignant neoplasms of genetic origin in Drosophila melanogaster. Science. 1978; 200(4349):1448-59. [PubMed: 96525]

19. Betschinger J, Mechtler K, Knoblich JA. Asymmetric segregation of the tumor suppressor brat regulates self-renewal in Drosophila neural stem cells. Cell. 2006; 124(6):1241-53. [PubMed: 16564014]

20. Bello B, Reichert H, Hirth F. The brain tumor gene negatively regulates neural progenitor cell proliferation in the larval central brain of Drosophila. Development. 2006; 133(14):2639-48. [PubMed: 16774999]

21. Bowman SK, Rolland V, Betschinger J, Kinsey KA, Emery G, Knoblich JA. The tumor suppressors Brat and Numb regulate transit-amplifying neuroblast lineages in Drosophila. Developmental cell. 2008; 14(4):535-46. [PubMed: 18342578]

22. Ito K, Carracedo A, Weiss D, Arai F, Ala U, Avigan DE, et al. A PML-PPAR-delta pathway for fatty acid oxidation regulates hematopoietic stem cell maintenance. Nature medicine. 2012; 18(9): $1350-8$.

23. Ito K, Bernardi R, Morotti A, Matsuoka S, Saglio G, Ikeda Y, et al. PML targeting eradicates quiescent leukaemia-initiating cells. Nature. 2008; 453(7198):1072-8. [PubMed: 18469801]

24. Kharas MG, Lengner CJ, Al-Shahrour F, Bullinger L, Ball B, Zaidi S, et al. Musashi-2 regulates normal hematopoiesis and promotes aggressive myeloid leukemia. Nature medicine. 2010; 16(8): 903-8.

25. Gomez-Lopez S, Lerner RG, Petritsch C. Asymmetric cell division of stem and progenitor cells during homeostasis and cancer. Cellular and molecular life sciences : CMLS. 2014; 71(4):575-97. [PubMed: 23771628]

26. Bieging KT, Mello SS, Attardi LD. Unravelling mechanisms of p53-mediated tumour suppression. Nature reviews Cancer. 2014; 14(5):359-70.

27. Spike BT, Wahl GM. p53, Stem Cells, and Reprogramming: Tumor Suppression beyond Guarding the Genome. Genes \& cancer. 2011; 2(4):404-19. [PubMed: 21779509]

28. Cicalese A, Bonizzi G, Pasi CE, Faretta M, Ronzoni S, Giulini B, et al. The tumor suppressor p53 regulates polarity of self-renewing divisions in mammary stem cells. Cell. 2009; 138(6):1083-95. [PubMed: 19766563]

29. Sugiarto S, Persson AI, Munoz EG, Waldhuber M, Lamagna C, Andor N, et al. Asymmetrydefective oligodendrocyte progenitors are glioma precursors. Cancer cell. 2011; 20(3):328-40. [PubMed: 21907924]

30. Suda T, Suda J, Ogawa M. Disparate differentiation in mouse hemopoietic colonies derived from paired progenitors. Proceedings of the National Academy of Sciences of the United States of America. 1984; 81(8):2520-4. [PubMed: 6585813]

31. Shen Q, Zhong W, Jan YN, Temple S. Asymmetric Numb distribution is critical for asymmetric cell division of mouse cerebral cortical stem cells and neuroblasts. Development. 2002; 129(20): 4843-53. [PubMed: 12361975]

32. Chen G, Kong J, Tucker-Burden C, Anand M, Rong Y, Rahman F, et al. Human Brat ortholog TRIM3 is a tumor suppressor that regulates asymmetric cell division in glioblastoma. Cancer research. 2014

33. He L, He X, Lim LP, de Stanchina E, Xuan Z, Liang Y, et al. A microRNA component of the p53 tumour suppressor network. Nature. 2007; 447(7148):1130-4. [PubMed: 17554337]

34. Bu P, Chen KY, Chen JH, Wang L, Walters J, Shin YJ, et al. A microRNA miR-34a-regulated bimodal switch targets Notch in colon cancer stem cells. Cell stem cell. 2013; 12(5):602-15. [PubMed: 23642368]

35. Colaluca IN, Tosoni D, Nuciforo P, Senic-Matuglia F, Galimberti V, Viale G, et al. NUMB controls p53 tumour suppressor activity. Nature. 2008; 451(7174):76-80. [PubMed: 18172499] 
36. Hwang WL, Jiang JK, Yang SH, Huang TS, Lan HY, Teng HW, et al. MicroRNA-146a directs the symmetric division of Snail-dominant colorectal cancer stem cells. Nature cell biology. 2014; 16(3):268-80. [PubMed: 24561623]

37. Lien WH, Fuchs E. Wnt some lose some: transcriptional governance of stem cells by Wnt/betacatenin signaling. Genes \& development. 2014; 28(14):1517-32. [PubMed: 25030692]

38. Dey-Guha I, Wolfer A, Yeh AC, J GA, Darp R, Leon E, et al. Asymmetric cancer cell division regulated by AKT. Proceedings of the National Academy of Sciences of the United States of America. 2011; 108(31):12845-50. [PubMed: 21757645]

39. Wicha MS, Liu S, Dontu G. Cancer stem cells: an old idea--a paradigm shift. Cancer research. 2006; 66(4):1883-90. discussion 95-6. [PubMed: 16488983]

40. Eyler CE, Rich JN. Survival of the fittest: cancer stem cells in therapeutic resistance and angiogenesis. Journal of clinical oncology : official journal of the American Society of Clinical Oncology. 2008; 26(17):2839-45. [PubMed: 18539962]

41. Kang Y, Ding M, Tian G, Guo H, Wan Y, Yao Z, et al. Overexpression of Numb suppresses tumor cell growth and enhances sensitivity to cisplatin in epithelioid malignant pleural mesothelioma. Oncology reports. 2013; 30(1):313-9. [PubMed: 23624653]

42. Blake W. "The Tyger". Songs of Innocence and Experience: Shewing the Two Contrary States of the Human Soul. 1794 


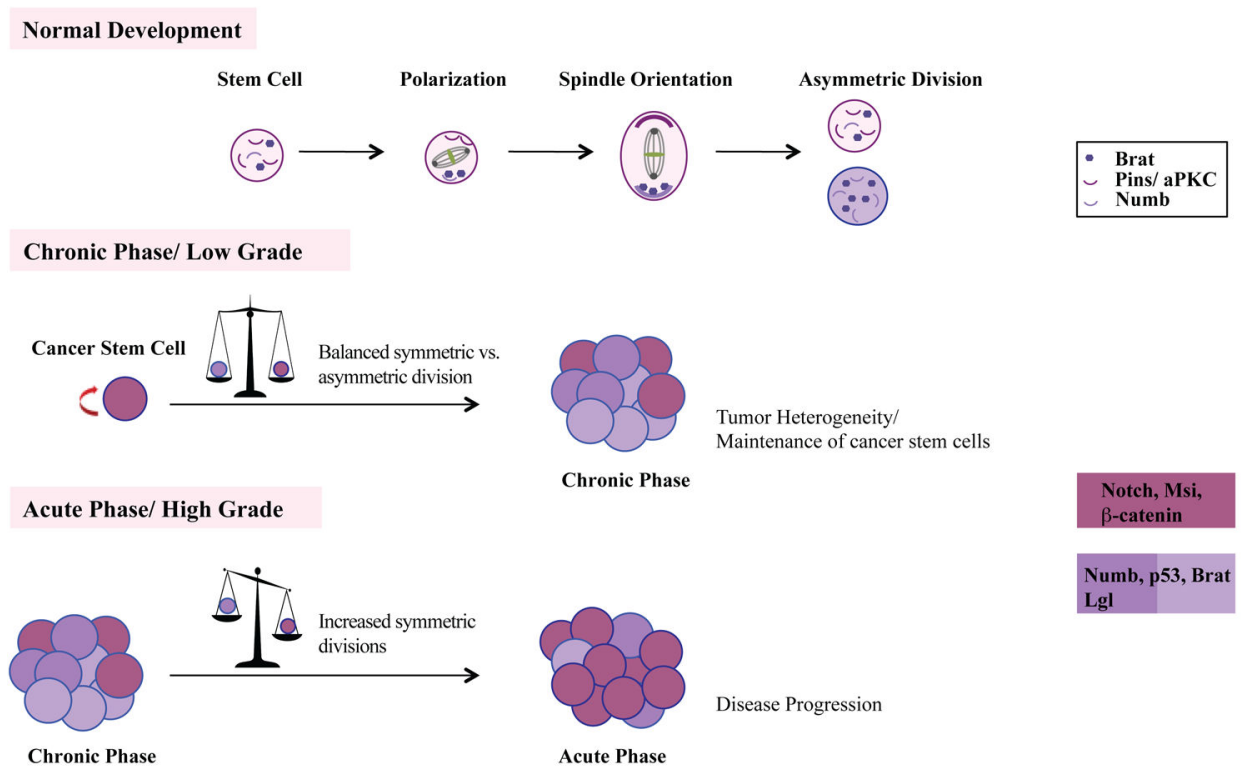

Basic motif of asymmetric division in normal development (top). Subversion of asymmetric division during oncogenesis to establish disease and create heterogeneity (middle), or promote disease progression (bottom).

Figure 1.

Top: Basic motif of asymmetric division in normal development. The apical localization of aPKC in the dividing stem cell promotes asymmetric distribution of fate determinants such as Numb and Brat. Subsequent inheritance of the fate determinants into one daughter directs a differentiated fate (purple).

Middle, Bottom: Subversion of asymmetric division during oncogenesis.

Balanced symmetric and asymmetric divisions allow maintenance of cancer stem cell populations and create heterogeneity during the chronic phase or low grade. In these cancers, the maintenance of Numb and p53 levels allows continued differentiation (middle). Accrual of additional mutations results in increased symmetric renewal divisions leading to a more undifferentiated and malignant state. This is associated with elevated expression of stem cell genes such as Notch, Msi and $\beta$-catenin (bottom). 